\title{
Prolonged Survival Using First-Line Pazopanib in a Filipino Male with Renal Cell Carcinoma and Brain Metastasis: A Case Report
}

\author{
Christianne V. Mojica ${ }^{a} \quad$ Gerard Vincent A. Aguas ${ }^{a}$ \\ Gerardo Thomas H. Cornelio $^{b}$ Ludwig F. Damian ${ }^{a}$ \\ anstitute for Neurosciences, St. Luke's Medical Center Quezon City and, Global City, \\ Philippines; ${ }^{b}$ Cancer Institute, St. Luke's Medical Center, Global City, Philippines
}

\section{Keywords}

Pazopanib $\cdot$ Tyrosine kinase inhibitor $\cdot$ Renal cell carcinoma $\cdot$ Brain metastasis

\begin{abstract}
Renal cell carcinoma is one of the leading causes of cancer worldwide. Brain metastasis is a poor prognostic factor among patients with this disease. The advancements in understanding of the molecular framework behind malignancy and brain metastasis led to more sophisticated treatment regimens which include targeted drugs and immunotherapy. While the role of tyrosine kinase inhibitors in metastatic renal cell carcinoma has been proven in the literature, its specific role among patients with brain metastasis has not yet been fully elucidated. We report a case of a Filipino male with renal cell carcinoma and brain metastasis who underwent stereotactic radiosurgery of his right frontal lesion followed by pazopanib taken initially at $800 \mathrm{mg} /$ day and then decreased to $600 \mathrm{mg} /$ day. A significant increase in creatinine level led to the discontinuation of the medication after $>3$ years. He had a remarkable progressionfree survival of 38 months. This is the first documented case of such significant response to pazopanib in a patient with renal cell carcinoma and brain metastasis. In the Philippine setting where options for cancer treatment are limited by the prohibitive cost of medications, this case can support the use of pazopanib as a potent agent for treating patients with this condition.
\end{abstract}

\section{Karger $\stackrel{2}{\circ}$}


Mojica et al.: Prolonged Survival Using First-Line Pazopanib

\section{Introduction}

Kidney cancer is the 14 th most common cancer worldwide. In the Philippines, a total of 2,384 new cases of kidney cancer were recorded in 2020. In the same year, it accounted for 1,229 cancer deaths [1]. The high burden of this disease is reflected in the 33,067 disability-adjusted life years associated with kidney cancer locally from 1990 to 2017 [2]. Renal cell carcinoma (RCC) is the most common type of kidney malignancy. In a recent single-center study, $60 \%$ of patients with RCC progressed to develop brain metastasis while as much as $29.1 \%$ of patients had brain metastasis at the initial diagnosis of cancer. Brain metastasis is associated with dismal outcome with a median overall survival of 8.4 months after diagnosis of brain metastasis [3]. Over the years, treatment options for brain metastasis have evolved to include immune checkpoint inhibitors and targeted therapy [4].

We report a case of Filipino male with metastatic RCC who underwent stereotactic radiosurgery (SRS) of his right frontal lobe lesion followed by pazopanib taken initially at $800 \mathrm{mg}$ / day and then decreased to $600 \mathrm{mg} /$ day. His disease was remarkably controlled for $>3$ years until pazopanib was stopped due to increasing level of creatinine. Progression of brain metastasis was noted immediately after the discontinuation of pazopanib.

\section{Case Report/Case Presentation}

The patient was initially diagnosed with RCC stage II presenting as gross hematuria. He subsequently underwent right radical nephrectomy in March 2011. He was asymptomatic in the interim until November 2017 when he started to have progressive weakness and numbness of the left upper and lower extremities. In January 2018, a contrast cranial MRI was done which revealed an enhancing nodule on the right frontal lobe with extensive perilesional edema (Fig. 1A). He underwent SRS of the right frontal lobe tumor on January 17, 2018, and was discharged stable.

In April 2018, a whole-body PET/CT scan revealed decrease in the size of the right frontal lobe tumor. However, a new enhancing lesion was seen in the left parietal lobe (Fig. 1B). There was also a finding of a hypermetabolic spiculated lung nodule in the right lower lobe and smaller nodules in both lungs. He underwent CT-guided percutaneous fine-needle aspiration biopsy of the right lung nodule. Biopsy and immunohistochemical stains (positive for PAX-8 and CD10 and negative for TTF-1, Napsin A, and p63) were consistent with metastatic RCC. He was then started on pazopanib $800 \mathrm{mg} /$ day. This dose was maintained for 3 months until it was decreased to $600 \mathrm{mg} /$ day due to generalized weakness and formation of blisters on the upper and lower extremities.

In June 2018, a repeat cranial MRI showed decrease in the size of the right frontal lobe lesion and surrounding edema (Fig. 1C). The left parietal lobe lesion appeared as a faint FLAIR subcentimeter hyperintensity (not in the picture). Chest CT scan showed regression in the spiculated right lower lobe lesion. The nodules in both lungs were stable also. Subsequent chest CT scans in August and October 2018 showed stable lung nodules. Serial abdominal CT scans were negative for tumor recurrence. The patient was compliant with pazopanib 600 $\mathrm{mg} /$ day, and he remained to be asymptomatic.

In December 2018, a repeat whole-body PET/CT showed stable enhancing lesions on the right frontal and left parietal lobes (Fig. 1D). The hypermetabolic right lung nodules showed significant interval decrease in metabolic activity. Serial chest CT scans in May and September 2019 showed stable nodules. The abdominal CT scans remained to have negative findings. Pazopanib was continued.

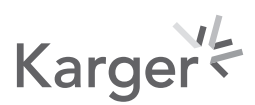




\section{Case Reports in Oncology}

\begin{tabular}{l|l}
\hline Case Rep Oncol 2021;14:1516-1521 \\
\hline DOI: 10.1159/000519745 & $\begin{array}{l}\text { @ 2021 The Author(s). Published by S. Karger AG, Basel } \\
\text { www.karger.com/cro }\end{array}$ \\
\hline
\end{tabular}

Mojica et al.: Prolonged Survival Using First-Line Pazopanib
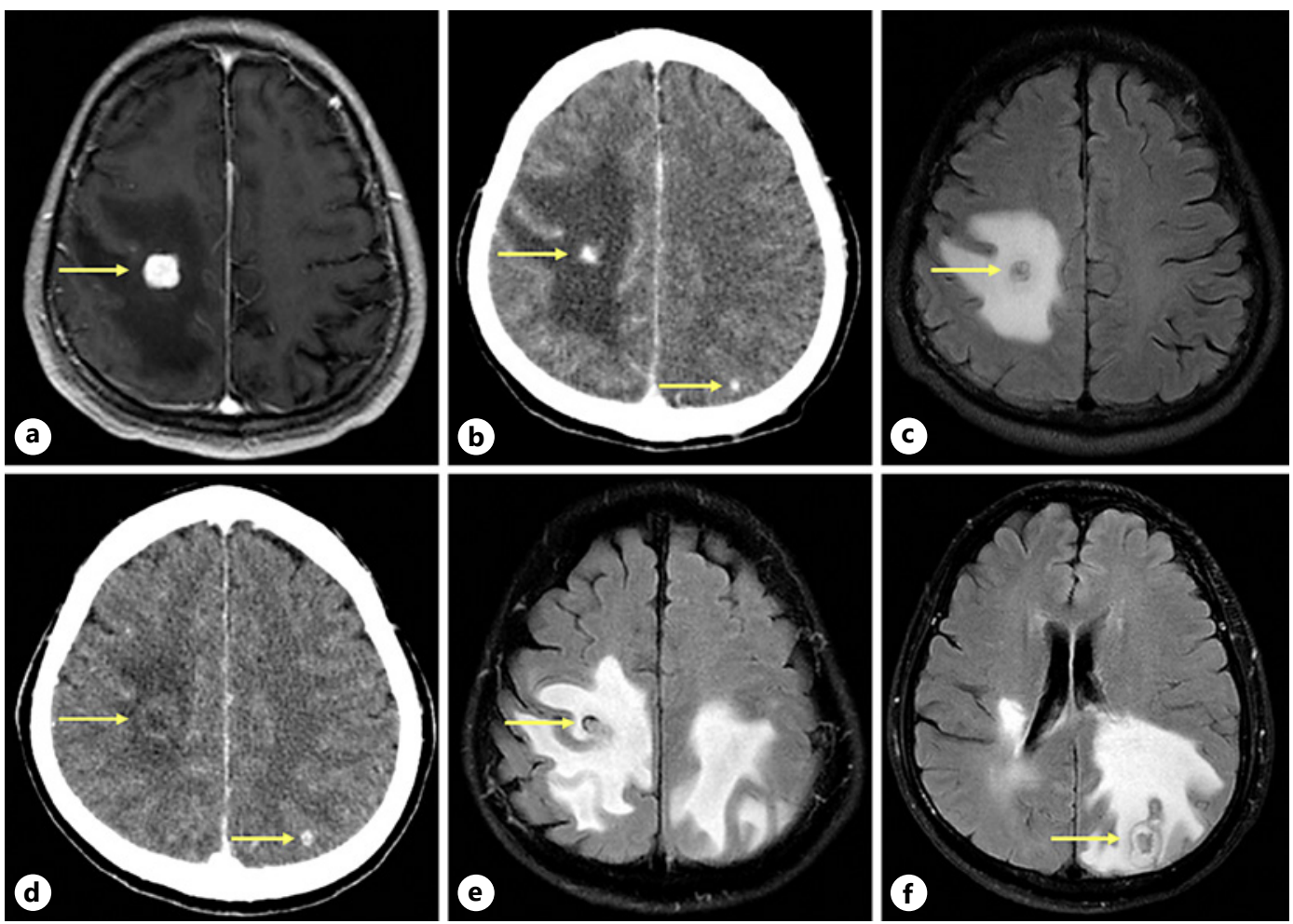

Fig. 1. Cranial MRI axial T1 contrast (a) image taken in January 2018 prior to SRS shows an enhancing ovoid lesion (yellow arrow) occupying the right frontal lobe measuring $1.5 \times 1.4 \mathrm{~cm}$ (APxT) with extensive perilesional vasogenic edema. Cranial contrast CT scan (b) done in April 2018 showed decrease in the size of the right frontal lobe mass $(0.9 \times 1 \mathrm{~cm})$ and a new enhancing lesion measuring $0.4 \times 0.3 \mathrm{~cm}$ in the left parietal lobe (yellow arrows). Follow-up MRI FLAIR (c) image taken in June 2018 after SRS and 2 months into pazopanib shows decrease in the size of the right frontal lesion $(0.7 \times 0.7 \mathrm{~cm})$ (yellow arrow) as well as the surrounding edema. Cranial contrast CT scan (d) done in December 2018 shows stable enhancing lesions on the right frontal and left parietal lobes (yellow arrows). Follow-up FLAIR (e, Ff) images taken in August 2021, 2 months after the last intake of pazopanib, shows the right frontal lobe lesion measuring $1.1 \times 0.8 \mathrm{~cm}$ and the left parietal lobe lesion measuring $1.8 \times 1.6 \mathrm{~cm}$ (yellow arrows) with extensive perilesional vasogenic edema.

In January 2021, he started to have increasing creatinine levels and was monitored closely. However, in June 2021, his creatinine elevated to $3.92 \mathrm{mg} / \mathrm{dL}$, and hence pazopanib was discontinued. He immediately noted progressive generalized body weakness after discontinuation of pazopanib. In August 2021, he presented with a 4-day history of left-sided weakness followed by blurring of vision. He was admitted on August 8, 2021. He was seen awake but with right homonymous hemianopsia and left-sided hemiparesis. Noncontrast cranial MRI showed increase in size of the left parietal lobe lesion while the frontal lobe lesion was relatively stable. There was significant perilesional edema (Fig. 1E, F). Chest CT scan findings were stable, and abdominal CT scan remained negative. He was started on dexamethasone $5 \mathrm{mg}$ IV every $6 \mathrm{~h}$ and levetiracetam $500 \mathrm{mg}$ twice daily as seizure prophylaxis. His condition improved significantly over the following days. He was offered to start pembrolizumab with lenvatinib, but due to cost, he decided to be given sunitinib $37.5 \mathrm{mg}$ daily instead. Dexamethasone was tapered down, and he was sent home with no visual field defects and with only minimal weakness of the left lower extremity. 


\section{Discussion/Conclusion}

Treatment for metastatic RCC includes multiple modalities to cover for both local and systemic recurrence. For brain metastasis, SRS has gained consideration over whole brain radiation therapy (WBRT) and even to surgery when the location of the lesion is not favorable. A recent study found that SRS resulted in local control of $92 \%$ at 1 year [5]. The patient underwent SRS of the right frontal lobe lesion which was located along the motor strip. There was decrease in the size of lesion on follow-up, but a new lesion on the left parietal lobe and the finding of lung nodules prompted to start systemic therapy.

In 2007, a phase III trial proved the efficacy of sunitinib over interferon alpha among patients with metastatic RCC. Unfortunately, patients with brain metastasis were excluded in the study [6]. Pazopanib, another tyrosine kinase inhibitor, was then studied among patients with metastatic RCC. The landmark trials COMPARZ and PISCES which compared pazopanib versus sunitinib were published in 2013 and 2014, respectively. The COMPARZ study found that pazopanib was noninferior to sunitinib in terms of efficacy. However, patients treated with pazopanib had better quality of life and developed less adverse effects [7]. The PISCES study looked into patient preference for either of the tyrosine kinase inhibitors and found that $70 \%$ of patients favored pazopanib over sunitinib. Again, the safety profile and quality of life were the considerations [8]. Both of these trials used pazopanib $800 \mathrm{mg}$ daily. Interestingly, both of these trials excluded patients with brain metastasis. To date, there has been no randomized controlled trial yet on the use of pazopanib on RCC and brain metastasis. Limited case reports and retrospective studies looked into pazopanib for such condition, but the results have not been this remarkable as seen in our patient.

One study reported a case of RCC in a 73-year-old female initially given sunitinib but then developed multiple brain metastasis. She then underwent WBRT which controlled the brain lesions initially. In the 6th month however, she had a relapse and pazopanib was started at $800 \mathrm{mg}$ daily. After 6 months, the dose was decreased to $600 \mathrm{mg}$ daily due to adverse effects until it was eventually discontinued. She developed progression of brain metastasis 11 months after [9]. Another case reported a 74-year-old male with RCC and brain metastasis at diagnosis who was initially given WBRT. Five months after WBRT, he developed extracranial disease progression and was initially given everolimus and then switched to pazopanib 800 mg daily due to poor response with everolimus. The brain metastasis was controlled until repeat cranial MRI showed progression of lesions in the 6th month of treatment [10].

A case series in China looked into the efficacy of first-line pazopanib at $800 \mathrm{mg}$ daily on 31 patients with metastatic RCC including 4 patients with brain metastasis. The median follow-up was 12.7 months, and the progression-free survival (PFS) was 10.1 months. Unfortunately, those with brain metastasis accounted for $42.9 \%$ of the occurrence of disease progression among these patients [11]. A retrospective study in Korea found that pazopanib had a similar efficacy with sunitinib among patients with metastatic RCC including those with lesions in the brain. The median follow-up was 16.4 months, and the PFS for pazopanib was 10.1 months. No subset analysis for those patients with brain metastasis was done [12].

This is the first documented case of such remarkable response to first-line pazopanib among patients with RCC and brain metastasis with a recorded PFS of 38 months. The disease was evidently controlled by pazopanib at a dose of $600 \mathrm{mg}$ daily which was even lower than the standard dose of $800 \mathrm{mg}$. It was evident that the discontinuation of pazopanib due to its adverse effects led to the progression of symptoms. Had there been no complication to kidney function which necessitated discontinuation of the drug, we believe that the brain and lung metastases of the patient would have remained to be controlled.

In the Philippine setting where options for cancer treatment can be limited by the prohibitive cost of medications, this case can support the potential use of pazopanib as a potent 
agent for treating patients with RCC and brain metastasis. Pazopanib is more economical than sunitinib in our local setting, and this may prove to be a more sustainable option for patients. This case further highlights the need for more research on the potential role of pazopanib among patients with RCC and brain metastasis.

\section{Statement of Ethics}

This study has been registered by the St. Luke's Institutional Ethics Review Committee and has been exempt from ethical review (August 2021). The patient gave written informed consent for the publication of this case report including the accompanying images.

\section{Conflict of Interest Statement}

The authors have no conflicts of interest to declare.

\section{Funding Sources}

This research did not receive any specific grant from funding agencies in the public, commercial, or not-for-profit sectors.

\section{Author Contributions}

Dr. Christianne V. Mojica is the main author of the manuscript and contributed to data collection. Dr. Gerard Vincent A. Aguas is a co-author in the manuscript and contributed to data collection. Dr. Gerardo Thomas H. Cornelio is a co-author in the manuscript and adviser in this study. Dr. Ludwig F. Damian is a co-author in the manuscript and adviser in this study.

\section{Data Availability Statement}

Inquiries regarding the access to the clinical history and imaging of the patient can be directed to the corresponding author.

\section{References}

1 Sung H, Ferlay J, Siegel R, Laversanne M, Soerjomataram I, Jemal A, et al. Global cancer statistics 2020: GLOBOCAN estimates of incidence and mortality worldwide for 36 cancers in 185 countries. CA Cancer J Clin. 2021;71:209-49.

2 Safiri S, Kolahi A, Mansourina M, Almasi-Hashiani A, Ashrafi-Asgarabad A, Sullman M, et al. The burden of kidney cancer and its attributable risk factors in 195 countries and territories, 1990-2017. Sci Rep. 2020;10:13862.

3 Suarez-sarmiento A, Nguyen K, Syed J, Nolte A, Ghabili K, Cheng M, et al. Brain metastasis from renal-ell carcinoma: an institutional study. Clin Genitourin Cancer. 2019;17(6):1163-70.

4 Matsui Y. Current multimodality treatments against brain metastases from renal cell carcinoma. Cancers. 2020;12:1-17.

5 Wardak Z, Christie A, Bowman A, Stojadinovic S, Nedzi L, Barnett S, et al. Stereotactic radiosurgery for multiple brain metastases from renal-cell carcinoma. Clin Genitourin Cancer. 2019;17(2):273-80.

6 Motzer RJ, Hutson TE, Tomczak P, Michaelson DM, Bukowski RM, Oudard S, et al. Overall survival and updated results for sunitinib compared with interferon alfa in patients with metastatic renal cell carcinoma. J Clin Oncol. 2009;27(22):3584-90.

\section{Karger's}


7 Motzer RJ, Hutson TE, Cella D, Reeves J, Hawkins R, Guo J, et al. Pazopanib versus sunitinib in metastatic renalcell carcinoma. N Engl J Med. 2013;369(8):722-31.

8 Escudier B, Porta C, Bono P, Powles T, Eisen T, Sternberg C, et al. Randomized, controlled, double-blind, crossover trial assessing treatment preference for pazopanib versus sunitinib in patients with metastatic renal cell carcinoma: PISCES Study. J Clin Oncol. 2014;32:1412-8.

9 Hingorani M, Dixit S, Maraveyas A. Pazopanib-induced regression of brain metastasis after whole brain palliative radiotherapy in metastatic renal cell cancer progressing on first-line sunitinib: a case report. World J Oncol. 2014;5(5-6):223-7.

10 Jacobs C, Kim DW, Straka C, Timmerman RD, Brugarolas J. Prolonged survival of a patient with papillary renal cell carcinoma and brain metastases using pazopanib. J Clin Oncol. 2013;31(7):e114-7.

11 Wang B, Song J, Chen H. First-line pazopanib treatment in metastatic renal cell carcinoma: real-world data from a single chinese center. Front Pharmacol. 2020;11:1-5.

12 Kim MS, Chung HS, Hwang EC, Jung SI, Kwon DD, Hwang JE, et al. Efficacy of first-line targeted therapy in realworld korean patients with metastatic renal cell carcinoma: focus on sunitinib and pazopanib. J Korean Med Sci. 2018;33(51):1-10. 